\title{
Robust decentralized power system controller design: Integrated approach
}

\author{
Vojtech Veselý
}

\begin{abstract}
A unique approach to the design of gain scheduled controller (GSC) is presented. The proposed design procedure is based on the Bellman-Lyapunov equation, guaranteed cost and robust stability conditions using the parameter dependent quadratic stability approach. The obtained feasible design procedures for robust GSC design are in the form of BMI with guaranteed convex stability conditions. The obtained design results and their properties are illustrated in the simultaneously design of controllers for simple model (6-order) turbogenerator. The results of the obtained design procedure are a PI automatic voltage regulator (AVR) for synchronous generator, a PI governor controller and a power system stabilizer for excitation system.
\end{abstract}

K e y w or d s: gain scheduled controller, Bellman-Lyapunov equation

\section{Introduction}

The main objective of power system control is to maintain a continuous supply of power with acceptable quality, to all consumers in the system. There are two basic controls:

- to achieve reactive power balance, acceptable voltage profile (automatic voltage control AVC), and

- real power balance-acceptable frequency values (automatic load frequency control) or automatic generation control.

The problem of Automatic Voltage Control and Automatic Load frequency control has been extensively studied in the last decade by a number of researchers and many control design procedures have been reported in literature, [1-16]. The obtained results can be summarized as follows. The objective of the paper [1] is to design nonlinear excitation controllers for single-machine infinite bus power systems. Nonlinear controllers are more effective in providing large stability margins than linear counterparts. The concept of feedback linearization is used. The proposed excitation controller [3] consists of a stabilizing PSS loop and voltage regulating loop. Certain connection between the two loop PSS-AVR schemes are discussed. In [4] a practical design of an intelligent type controller using polynomial neural network is explored. The obtained computer simulation results demonstrate clearly that the performance of the developed controllers offers competitive damping effects on the generator oscillations. The papers $[5,24]$ proposes a nonlinear robust controller for steam governor control in power systems. The analytical synthesis problem of a coordinating regulator for power system consisting of turbogenerators is considered in [6]. A review of the power system stabilizer design methods is considered in [7]. In [8] a new multi-objective function as an optimization problem is proposed for the coordination of power system stabilizer and static synchronous series compensator. In [9] proposes decentralized excitation controllers. Compared with conventional excitation controllers a parameter adaptation scheme is proposed to improve the transient stability of the closed loop system. The papers $[10,25]$ propose optimal and a novel Lyapunov-based excitation control technique for multimachine power system. A completely controllable linear system is constructed to design the excitation decentralized controller. The papers $[11,23,25]$ provide a detailed account to determine the parameters of the power system stabilizer using a small signal approach. To tackle the uncertainty problem, the paper [13] focuses on a new robust decentralized control design of robust PSS. The conventional lead/lag PSS which are designed without considering uncertainty may deteriorate a system stability. [14] deals with the frequency domain design of a robust power system stabilizer using the equivalent subsystem method.

The Global Asymptotic Stability (GAS) AVR and power regulation [15] are of critical importance for power security. However, the simultaneous design of AVR and governor control has not been achieved. In the above paper a completely controllable linear system is used for both AVR and power regulation for control system design. The paper [16] presents a novel procedure for the design of PSS using the linear quadratic regulator approach. To model the power system one can use the excellent books [17-19].

The above short observation implies:

- power system belongs to class of highly nonlinear systems and therefore it demands use of corresponding controller design procedure;

- GAS automatic voltage regulation and governor control are of critical importance for power system security;

Faculty of Electrical Engineering and Information Technology, Slovak University of Technology, Ilkovičova 3, 81219 Bratislava, Slovakia vojtech.vesely@stuba.sk 
- design procedures to determine the controller parameters of excitation and governor controller on the main stream in the references base on the linearized model of the power system;

- when the nonlinear model of power system is used for controller parameters design the Lyapunov function based approach is preferred or other class of method of intelligent control.

In this paper we pursue the idea of using an integrated approach to the design of AVR, governor control and PSS simultaneously for the nonlinear model of power system. The power system nonlinear model first we transform to the linear-parameter-varying (LPV) system, and then for controller parameters design in this paper a proposed novel gain scheduling approach [20] with convex stability conditions has been adapted.

\section{Preliminaries and problem formulation}

In this section we transform the nonlinear model of turbogenerator to the LPV system. Under the well-known assumptions [17-19] a model of turbogenerator which consists of synchronous generator (SG) and thermal turbine can be described as follows.

- Third order model of synchronous generator $\left(E_{q}, \delta, \omega\right)$ in p.u.

$$
\begin{gathered}
U_{q}=I_{d} X_{d}+E_{q}-I_{q} R_{a}, \\
U_{d}=-I_{q} X_{q}-I_{d} R_{a} \\
U_{b} k_{G 0}=E_{q}+T_{d 0}^{\prime} \frac{\mathrm{d} E_{q}}{\mathrm{~d} t}+T_{d 0}^{\prime} \frac{\mathrm{d} I_{d}}{\mathrm{~d} t}\left(X_{d}-X_{d}^{\prime}\right), \\
T_{j} \frac{\mathrm{d} \omega}{\mathrm{d} t}=P_{T}-P_{e}, \\
P_{e}=P+P_{a s}, \quad P_{a s} \doteq D \omega \\
P=E_{q} I_{q}+I_{d} I_{q}\left(X_{d}-X_{q}\right)-R_{a}\left(I_{d}^{2}+I_{q}^{2}\right)
\end{gathered}
$$

where $I_{d}, I_{q}$ - currents flowing in the fictitious $d$ and $q$ axis armature coils,

$E_{q}-q$-axis component of the internal emf, proportional to the field current, excitation current of SG,

$U_{q}, U_{d}$ - voltages across the fictitious $d$ and $q$ axis armature coils,

$P_{e}$ - total electric power generated by SG to the system,

$P_{a s}$-damping power,

$U_{b}$ - input voltage applied to the field winding,

$T_{d 0}^{\prime}$ - open-circuit $d$-axis transient time constant,

$T_{j}$ - inertia coefficient of turbogenerator,

$X_{d}, X_{q}, X_{d}^{\prime}$ - reactance and transient reactance of the fictitious $d$ and $q$ axis armature windings,

$R_{a}$ - resistance of the armature winding of a $\mathrm{SG}$,

$\left.U=\sqrt{(} U_{d}^{2}+U_{q}^{2}\right)$ - voltage of the generator terminals.

The time derivative of the rotor load angle $\delta$

$$
\frac{\mathrm{d} \delta}{\mathrm{d} t}=\Delta \omega=\omega-\omega_{s}
$$

is the rotor speed deviation in $\mathrm{rad} / \mathrm{s}$ and $\omega_{s}-$ power system angular speed.
In the next section the following denotation will used $\triangle \omega=\omega$

Thermal turbine model with governor valve in simplified structure [19] is given by the following third order transfer function

$$
G_{T(s)}=\frac{P_{T(s)}}{P_{R(s)}}=\frac{s b_{1}+b_{0}}{s^{3} a_{3}+s^{2} a_{2}+s a_{1}+1}
$$

where $P_{T(s)}$ - output of turbine power,

$P_{R(s)}$ - output of turbine power controller,

$$
\begin{gathered}
b_{1}=k_{l} T_{h}+k_{h} T_{l}, \quad b_{0}=k_{l}+k_{h}=1, \quad a_{3}=T_{s} T_{l} T_{h}, \\
a_{2}=T_{l} T_{h}+T_{s}\left(T_{l}+T_{h}\right), \quad a_{1}=T_{l}+T_{h}+T_{s} .
\end{gathered}
$$

Typical values of the parameters are:

servomotor time constant $T_{s}=0.1 \mathrm{~s}$, low pressure gain and time constant $k_{l}=0.7 \mathrm{pu}, T_{l}=4-11 \mathrm{~s}$, high pressure gain and time constant $k_{h}=0.3 \mathrm{pu}, T_{h}=0.1 \mathrm{~s}$.

In general, turbogenerators in power system are always subject to different disturbances, eg periodic load variation, swings of the other turbogenerators in transient state, and so on. In order to take account of these disturbances and determine the stability of such systems in [27] the authors introduced the One-Machine-to-QuasiInfinite Bus System. In the following we will study a single machine connected to a large power system through transmission lines. We will assume that a large power system belong to the class of infinite bus system with bus $\{$ voltage, angular speed $\}=\left(U_{s}, \omega_{s}\right)[18,19]$. Because of the relative size of the power system to which the machine is supplying power, the dynamics associated with the machine will cause virtually no change in the voltage $U_{s}$ and frequency $\omega_{s}$. To relaxed the infinite bus model (or made our obtained results useful for multimachine power system model) we are assuming that some system variables are unknown and lying within two values. Transmission lines can be transformed to the $\mathrm{T}$ equivalent circuit with impedance $\bar{z}_{1}, \bar{z}_{2}$, and reluctant impedance $\bar{z}_{3}$. Using Kirchhoff's laws for currents on the $d$ and $q$ axis one can obtain as follows.

$$
\begin{aligned}
& I_{d}=-\frac{E_{q}}{M}+\frac{U_{s}}{z_{12}\left(1+\frac{X_{d}-X_{q}}{z_{11}} \sin \varphi_{11}\right)} \sin \left(\delta+\varphi_{12}\right) \\
& I_{q}=\frac{E_{q}}{M \tan \varphi_{11}}+\frac{U_{s}}{z_{12}} \times \\
& \sqrt{1+\left(\frac{\left(X_{d}-X_{q}\right) \cos \varphi_{11}}{\left.z_{11}+\left(X_{d}-X_{q}\right) \sin \varphi_{11}\right)}\right)^{2}} \sin \left(\delta+\varphi_{12}-\psi\right)
\end{aligned}
$$

where

$$
\begin{gathered}
M=\frac{z_{11}}{\sin \varphi_{11}}+X_{d}-X_{q} \\
\psi=\arctan \frac{z_{11}+\left(X_{d}-X_{q}\right) \sin \varphi_{11}}{\left(X_{d}-X_{q}\right) \cos \varphi_{11}}, \\
\bar{z}_{11}=j X_{q}+\bar{z}_{1}+\frac{\bar{z}_{2} \bar{z}_{3}}{\bar{z}_{2}+\bar{z}_{3}}=z_{11} e^{j \varphi_{11}}, \\
\bar{z}_{12}=j X_{q}+\bar{z}_{1}+\frac{\bar{z}_{2}}{\bar{z}_{3}}\left(\bar{z}_{3}+\bar{z}_{1}+j X_{q}\right)=z_{12} e^{j \varphi_{12}}
\end{gathered}
$$




\subsection{Linear parameter varying model of turbogenerator}

Now, we are ready to transform (1), (2) and (3) to the linear parameter varying system. For more detail how to obtain LPV model for nonlinear system the reader can consult the survey paper [28]. The gain scheduled model of turbogenerator is obtain using the following steps.

1. Choose the gain scheduling variable, which captures parametric dependence of the nonlinear plant model, in our case we will choose it as the electrical power $P$ pu.

2. Choose working points where LPV model and nonlinear model have to be equal $(w=1,2,3, P=$ $(0.3,0.7,1) \mathrm{pu})$. For chosen three working points one need to have $p=2$ gain scheduling parameters

3. For robust controller design, choose the uncertain parameters. To relaxed the infinite bus model (or to made ours results useful also in case of multimachine power system model) we are assuming that the values of system voltage $U_{s}$ and frequency is unknown and lying within a given two values, (for our case $k=2, U_{s}$ and instead of frequency we will use the reluctant inductance $X_{v 3}$ ) that is one assume that for uncertain parameters the following holds $U_{s} \in\left\langle U_{\text {smin }}, U_{\text {smax }}\right\rangle$ and $\bar{z}_{3}=j X_{v 3}$, $X_{v 3} \in\left\langle x_{v 3 \min }, x_{v 3 \max }\right\rangle$

4. At each chosen working points and two uncertain parameters, one builds a four $\left(2^{k}=4\right.$ linearized model of turbogenerator in the form

$$
\dot{x}=\bar{A}_{w i} x+\bar{B}_{w i} u, w=1,2,3, i=1,2,3,4, y=C x
$$

where $x(t) \in R^{n}$ is the state vector, $u(t) \in R^{m}$ is the control vector, $y(t) \in R^{l}$ is the output vector of system to be controlled.

5. Introduce LPV - gain scheduled model with polytopic uncertainties. (Let gain scheduled varying vector parameter is a function of electrical power $\theta=f(P)$ with $p=2$ gain scheduling variables),

$$
\begin{array}{r}
\dot{x}=A(\theta, \xi) x+B(\theta, \xi) u, \quad y=C x \\
A(\theta, \xi)=A_{0}(\xi)+\sum_{j=1}^{p} A_{j}(\xi) \theta_{j} \in R^{n \times n}, \\
A_{j}(\xi)=\sum_{i=1}^{2^{k}} A_{j i} \xi_{i}, \quad B(\theta, \xi) \in R^{n \times m}, \\
u=\left[\triangle U_{b} \quad \triangle P_{R}\right]^{\top}, \quad y=\left[\begin{array}{ll}
\triangle \times n & \triangle P
\end{array}\right]^{\top}
\end{array}
$$

In (7) $\xi \geq 0$ is constant or time varying unknown variable which satisfies $\sum_{i=1}^{2^{k}} \xi_{i}=1$ and $\theta \in R^{p}$ is a vector of known constant or time varying real gain-scheduled parameters. We assume that both lower and upper bounds are available for these parameters value and variation rates.

Specifically

- Each parameter $\theta_{j}, j=1,2, \ldots, p$ ranges between known extremal values,

$$
\theta_{j} \in \Omega_{\theta}:=\left\{\theta_{j} \in\left\langle\underline{\theta_{j}}, \overline{\theta_{j}}\right\rangle, j=1,2, \ldots, p .\right.
$$

- The rate of variation $\dot{\theta}_{j}$ is well defined at all times and satisfies

$$
\dot{\theta}_{j} \in \Omega_{t}:=\left\{\dot{\theta}_{j} \in\left\langle\underline{\theta_{j}}, \overline{\theta_{j}}\right\rangle, j=1,2, \ldots, p .\right.
$$

6 . For each uncertainties $i=1,2,3,4$ and all working points $w=1,2,3$ substitute extremal values of gainscheduled parameters to (7). We assume that extremal values are $\theta_{j}=-1$ or $\theta_{j}=1$ for $j=1,2$. Note that in our case $\theta_{1}=-1$ and $\theta_{2}=1$ does not exist. Compare the obtained results with (6). For each $i$ and working points $w=1,2,3$ one has got

$$
\begin{gathered}
\bar{A}_{1, i}=A_{0 i}-A_{1 i}-A_{2 i}, \theta_{1}=-1, \theta_{2}=-1, w=1, \\
\bar{A}_{2 i}=A_{0 i}+A_{1 i}-A_{2 i}, \theta_{1}=1, \theta_{2}=-1, w=2, \\
\bar{A}_{3 i}=A_{0 i}+A_{1 i}+A_{2 i}, \theta_{1}=1, \theta_{2}=1, w=3 .
\end{gathered}
$$

From (8) one obtains the simple equation for calculation of gain scheduled model for $i$-uncertainty

$$
\left[\begin{array}{ccc}
I & -I & -I \\
I & I & -I \\
I & I & I
\end{array}\right]\left[\begin{array}{c}
A_{0 i} \\
A_{1 i} \\
A_{2 i}
\end{array}\right]=\left[\begin{array}{c}
\bar{A}_{1 i} \\
\bar{A}_{2 i} \\
\bar{A}_{3 i}
\end{array}\right]
$$

7. For each working point $w=1,2,3$ one obtain 4 matrices $A_{w i}$ which lying in the polytope vertices of uncertain gain scheduled model of turbogenerator.

For I-part gain scheduled controller design the states of original system (7) need to be extended, for more detail see [21]. The control algorithm for PID controller is

$$
u(t)=K_{p} y(t)+K_{i} \int_{0}^{t} C x(\tau) \mathrm{d} \tau+K_{d} \dot{y}(t) .
$$

Integral term can be included into the state vector in the way defining the auxiliary state $z=\int_{0}^{t} x(\tau) \mathrm{d} \tau$ and PID control algorithm is

$$
u(t)=\left[\begin{array}{ll}
K_{p} & K_{i}
\end{array}\right] C\left[\begin{array}{c}
x(t) \\
z(t)
\end{array}\right]+\left[\begin{array}{ll}
K_{d} C_{d} & 0
\end{array}\right]\left[\begin{array}{c}
\dot{x}(t) \\
\dot{z}(t)
\end{array}\right]
$$

With controller integral term the new state and output vectors are given as $X(t)=\left[x(t)^{\top}, z(t)^{\top}\right]^{\top}, Y(t)=$ $\left[y(t)^{\top},(C z(t))^{\top}\right]^{\top}$, for other type of controller the plant state and output do not changes. In the next, for all type of controller, we will assume that without changing the denotation of state and output vectors $x(t)=X(t)$, $y(t)=Y(t)$, matrices and matrices dimensions the static output feedback control algorithm can provide the proportional and integral parts of the designed robust gain scheduled PID controller using (7).

The following problem is studied in this paper. Design a robust output feedback gain scheduled PID controller with control algorithm

$$
u=K(\theta) y+K_{d}(\theta) \dot{y}=K(\theta) C x+K_{d}(\theta) C_{d} \dot{x}
$$


where $K(\theta)=K_{0}+\sum_{j=1}^{p} K_{j} \theta_{j}=\left[K_{p}(\theta) \quad K_{i}(\theta)\right] \in$ $R^{m \times 2 l}, K_{d}(\theta)=K_{d 0}+\sum_{j=1}^{p} K_{d j} \theta_{j} \in R^{m \times l}$. For more detail to obtained new plant model with PID controller see [21]. $C_{d} \in R^{l \times n}$ is the SG output matrix for the derivative part of controller such that the controller ensures robust parameter dependent quadratic stability and guaranteed cost with respect to the closed loop system, $(7)+(12)$. To assess the performance quality, the quadratic cost function is used

$$
\begin{gathered}
J_{c}=\int_{t_{0}}^{\infty} J(x, u, \dot{x}, \theta) \mathrm{d} t \\
J(x, u, \dot{x}, \theta)=x^{\top} Q(\theta) x+u^{\top} R u+\dot{x}^{\top} S(\theta) \dot{x} \\
Q(\theta)=Q_{0}+\sum_{j=1}^{p} Q_{j} \theta_{j} \in R^{n \times n} \\
S(\theta)=S_{0}+\sum_{j=1}^{p} S_{j} \theta_{j} \in R^{n \times n}
\end{gathered}
$$

matrices are positive definite (semidefinite) and $R \in$ $R^{m \times m}$ is positive definite matrix.

Lemma 1. Consider the system (7) with control algorithm (10). Control algorithm (10) is the guaranteed cost control law for a closed loop system if and only if there is a Lyapunov function $V(\theta, \xi)$, such that the following condition holds:

$$
B_{e}(\theta, \xi)=\max _{u}\left(\frac{d V(\theta, \xi)}{d t}+J(x, u, \dot{x}, \theta) \leq 0 .\right.
$$

Equation (14) is known as the Bellman-Lyapunov equation and function $V(\theta, \xi)$ which satisfies (14) is the Lyapunov function. For a particular structure of the Lyapunov function $V(\theta, \xi)$ the obtained gain scheduled design procedure reduces from "if and only if" to "if".

\section{Design of gain scheduled PID controller}

This section formulates the theoretical approach to the robust PID gain-scheduled controller design with convex stability conditions for uncertain polytopic system (7). This ensures closed-loop system parameter dependent quadratic stability (PDQS) and guaranteed cost for all uncertain plant parameters $\Pi \in \Omega$, gain scheduled parameters $\theta \in \Omega_{\theta}$ and $\dot{\theta} \in \Omega_{t}$. Assume that in (14) the Lyapunov function is in the form

$$
\begin{aligned}
& V(x, \theta, \xi)=x^{\top} P(\theta, \xi) x \\
& P(\theta, \xi)=P_{0}(\xi)+\sum_{j=1}^{p} P_{j}(\xi) \theta_{j}, \\
& P_{j}(\xi)=\sum_{i=1}^{N} P_{j i} \xi_{i}, j=0,1,2, \ldots, p, N=2^{k} .
\end{aligned}
$$

The time derivative of $(15)$ is

$$
\begin{gathered}
\frac{\mathrm{d} V(\cdot)}{\mathrm{d} t}=\dot{x}^{\top} P(\theta, \xi) x+x^{\top} P(\dot{\theta}, \xi) x+x^{\top} P(\theta, \xi) \dot{x}= \\
{\left[\begin{array}{ccc}
\dot{x}^{\top} & x^{\top} & u^{\top}
\end{array}\right]\left[\begin{array}{ccc}
0 & P(\theta, \xi) & 0 \\
P(\theta, \xi) & P(\dot{\theta}, \xi) & 0 \\
0 & 0 & 0
\end{array}\right]\left[\begin{array}{l}
\dot{x} \\
x \\
u
\end{array}\right]} \\
P(\dot{\theta}, \xi)=\sum_{j=1}^{p} P_{j}(\xi) \dot{\theta}_{j} \leq \sum_{j=1}^{p} P_{j}(\xi) \rho_{j}
\end{gathered}
$$

assuming that $P_{j}(\xi) \geq 0$ and $\max \left|\dot{\theta}_{j}\right| \leq \rho_{j}>0$. Note that for the case of PDQS it is assumed that uncertain parameters are constants but LPV model system parameters can be changed by rate determined of $\dot{\theta}$. For the case of quadratic stability (QS) the rate of uncertain parameters changes may reach any values. For this case in (15) the Lyapunov matrix changes to

$$
P(\theta)=P_{0}+\sum_{j=1}^{p} P_{j} \theta_{j}
$$

In the next developments we will assume that PDQS approach is used. To separate matrix $P(\cdot)$ from matrices $A(\cdot), B(\cdot)$ introduce $N_{i} \in R^{n \times n}, i=1,2 ; N_{3} \in R^{n \times m}$; $N_{i} \in R^{m \times n}, i=4,5 ; N_{6} \in R^{m \times m}$ auxiliary matrices of corresponding dimensions in the following form

1) $2\left(N_{1} \dot{x}+N_{2} x+N_{3} u\right)^{\top}(\dot{x}-A(\theta, \xi) x-B(\theta, \xi) u)=0$,

2) $2\left(N_{4} \dot{x}+N_{5} x+N_{6} u\right)^{\top}\left(u-K(\theta) C x-K_{d}(\theta) C_{d} \dot{x}\right)=0$.

Summarizing (17) and (16) for the time derivative of the Lyapunov function one obtains

$$
\dot{V}(x, \theta, \xi)=z^{\top} W(\theta, \xi) z
$$

where $z^{\top}=\left[\begin{array}{lll}\dot{x}^{\top} & x^{\top} & u^{\top}\end{array}\right]$ and $W(\theta, \xi)=\left\{w_{i j}(\theta, \xi)\right\}_{3 \times 3}$ and

$$
\begin{gathered}
w_{11}(\theta, \xi)=N_{1}^{\top}+N_{1}-N_{4}^{\top} K_{d}(\theta) C_{d}-C_{d}^{\top} K_{d}(\theta)^{\top} N_{4}, \\
w_{12}(\cdot)=-N_{1}^{\top} A(\theta, \xi)+N_{2}+P(\theta, \xi)-N_{4}^{\top} K(\theta) C- \\
C_{d}^{\top} K_{d}(\theta)^{\top} N_{5}, \\
w_{13}(\cdot)=-N_{1}^{\top} B(\theta, \xi)+N_{3}+N_{4}^{\top}-C_{d}^{\top} K_{d}(\theta)^{\top} N_{6}, \\
w_{22}(\cdot)=-N_{2}^{\top} A(\theta, \xi)-A(\theta, \xi)^{\top} N_{2}+P(\dot{\theta}, \xi)- \\
N_{5}^{\top} K(\theta) C-C^{\top} K(\theta)^{\top} N_{5}, \\
w_{23}(\cdot)=-N_{2}^{\top} B(\theta, \xi)-A^{\top}(\theta, \xi) N_{3}+N_{5}^{\top}- \\
C^{\top} K(\theta)^{\top} N_{6}, \\
w_{33}(\cdot)=-N_{3}^{\top} B(\theta, \xi)-B(\theta, \xi)^{\top} N_{3}+N_{6}^{\top}+N_{6} .
\end{gathered}
$$

System (7) with control algorithm (12) is parameterdependent quadratically stable for $\theta \in \Omega_{\theta}, \dot{\theta} \in \Omega_{t}$ and uncertainty $\xi$ if the matrix $W(\theta, \xi)$ is negative definite 
(semidefinite). To obtain the guaranteed cost control algorithm substitute the time derivative of the Lyapunov function (16) and performance index (13) to the BellmanLyapunov equation (14)

$$
B_{e}=\dot{V}(x, \theta, \xi)+J(x, u, \dot{x}, \theta)=z^{\top} H(\theta, \xi) z \leq 0
$$

where $H(\theta, \xi)=\left\{h_{i j}(\theta, \xi)\right\}_{3 \times 3}$ and

$$
\begin{gathered}
h_{11}(\theta, \xi)=w_{11}(\theta, \xi)+S(\theta), \quad h_{12}(\theta, \xi)=w_{12}(\theta, \xi), \\
h_{13}(\theta, \xi)=w_{13}(\theta, \xi), \quad h_{22}(\theta, \xi)=w_{22}(\theta, \xi)+Q(\theta), \\
h_{23}(\theta, \xi)=w_{23}(\theta, \xi), \quad h_{33}(\theta, \xi)=w_{33}(\theta, \xi)+R .
\end{gathered}
$$

Matrix $H(\theta, \xi)$ is linear with respect to the gain scheduled parameter $\theta$ and uncertainty $\xi$ therefore $H(\theta, \xi)$ can be split into

$$
H(\theta, \xi)=H_{0}(\xi)+\sum_{j=1}^{p} H_{j}(\xi) \theta_{j}=\sum_{i=1}^{N}\left(H_{0 i}+\sum_{j=1}^{p} H_{i j} \theta_{j}\right) \xi_{i}
$$

The main results of the gain scheduled PID controller design, which ensure robust parameter-dependent quadratic stability and guaranteed cost, are given in the next theorem.

\section{THEOREM 1.}

The uncertain system (7) with PID gain scheduled controller (12) is a robust parameter dependent quadratically stable with guaranteed cost if there exists a positive definite matrix $P(\theta, \xi) \in R^{n \times n}$, matrices $N_{i}, i=1,2, \ldots, 6$, $Q(\theta), S(\theta)$, and $R$ such that

$$
\begin{gathered}
H_{0 i}+\sum_{j=1}^{p} H_{i j} \theta_{j} \leq 0, \\
i=1,2, \ldots, N ; \quad \theta \in \Omega_{\theta} ; \quad \dot{\theta} \in \Omega_{t}
\end{gathered}
$$

where $H_{0 i}=\left\{h_{0 i k l}\right\}_{3 \times 3}$,

$$
\begin{gathered}
h_{0 i 11}=N_{1}^{\top}+N_{1}-N_{4}^{\top} K_{d 0}^{\top} C_{d}-C_{d}^{\top} K_{d 0}^{\top} N_{4}+S_{0}, \\
h_{0 i 12}=-N_{1}^{\top} A_{0 i}+N_{2}+P_{0 i}-N_{4}^{\top} K_{0} C-C_{d}^{\top} K_{d 0}^{\top} N_{5}, \\
h_{0 i 13}=-N_{1}^{\top} B_{0 i}+N_{3}+N_{4}^{\top}-C_{d} K_{d 0}^{\top} N_{6}, \\
h_{0 i 22}=-N_{2}^{\top} A_{0 i}-A_{0 i}^{\top} N_{2}+\sum_{j=1}^{p} P_{j i} \rho_{j}- \\
N_{5}^{\top} K_{0} C-C^{\top} K_{0}^{\top} N_{5}+Q_{0}, \\
h_{0 i 23}=-N_{2}^{\top} B_{0 i}-A_{0 i}^{\top} N_{3}+N_{5}^{\top}-C^{\top} K_{0}^{\top} N_{6}, \\
h_{0 i 33}=-N_{3}^{\top} B_{0 i}-B_{0 i}^{\top} N_{3}+N_{6}^{\top}+N_{6}+R, \\
\left.h_{k l i j}\right\}_{3 \times 3}, \\
h_{11 i j}=-N_{4}^{\top} K_{d j} C_{d}-C_{d}^{\top} K_{d j}^{\top} N_{4}+S_{j}, \\
h_{12 i j}=-N_{1}^{\top} A_{i j}+P_{i j}-N_{4}^{\top} K_{j} C-C_{d}^{\top} K_{d j}^{\top} N_{5}, \\
h_{13 i j}=-N_{1}^{\top} B_{i j}-C_{d}^{\top} k_{d j}^{\top} N_{6}, \\
h_{22 i j}=-N_{2}^{\top} A_{i j}-A_{i j}^{\top} N_{2}-N_{5}^{\top} K_{j} C-C^{\top} K_{j}^{\top} N_{5}+Q_{j}, \\
h_{23 i j}=-N_{2}^{\top} B_{i j}-A_{i j}^{\top} N_{3}-C^{\top} K_{j}^{\top} N_{6}, \\
h_{33 i j}=-N_{3}^{\top} B_{i j}-B_{i j}^{\top} N_{3} .
\end{gathered}
$$

Note that inequality (21) is convex with respect to the gain scheduled parameter $\theta$ and uncertain parameter $\xi$, therefore (21) holds if and only if it is negative definite (semidefinite) for all $i=1,2, \ldots, N$ ( $\xi$-vertices) and $j=1,2, \ldots, p$ ( $\theta$-vertices $)$. Proof of theorem sufficient robust stability condition is based on the Lemma 1 , and equations (15), (16) and (17). Note that for calculation of feasible robust gain scheduled controller the designer need to insert to a program some values of variables. One of these values is the maximal value of rate to disturbance changes (in our case $\varrho_{i}=10 \mathrm{pu} / \mathrm{s}$ If the inequalities (bilinear matrix inequalities) BMI (21) are feasible with the given data the obtained PID gain scheduled controller ensure the parameter dependent quadratic stability and guaranteed cost for all defined regime of turbogenerator and disturbance rate.

\section{Example}

For the purpose of demonstrating the advantages of the proposed method we will use the case of a simple turbogenerator connected to a large-scale power system trough transmission lines. Because of the relative size of a large scale power system to which the turbogenerator is supplying power, the dynamics associated with the machine will cause virtually no change in the system voltage $U_{s}$ and frequency $\omega_{s}$. In general, generators in power systems are always subject to periodic or other type disturbances, eg periodic load variations, swings of the other generators in transient state, and so on, [27]. For take account above disturbances and guarantee the stability of closed-loop systems one can introduce the uncertainties to model of turbogenerator. We will assume that the system voltage is unknown and lying within two values $U_{s} \in\langle 0.9,1.1\rangle \mathrm{pu}$ and the second uncertainty to model above disturbances can be line reactance, which lying $X_{v 3} \in\langle 0.2,2\rangle \mathrm{pu}$. If we take the system frequency as a unknown variable the calculation load is very increased because when frequency is changed then all reactance of our model changes too (lines, SG and other parameters). Above two uncertainties relax the infinite bus model and made our obtained result more useful for multimachine power system model. Parameters of SG and transmission lines are as follows.

$$
\begin{aligned}
& T_{j}=0.02245 \mathrm{~s}^{2}, T_{d 0}^{\prime}=0.4 \mathrm{~s}, X_{d}=2 \mathrm{pu}, X_{d}^{\prime}=0.247 \mathrm{pu}, \\
& X_{q}=1.75 \mathrm{pu}, R_{a}=0, X_{v 1}=0.127 \mathrm{pu}, X_{v 2}=0.12 \mathrm{pu} .
\end{aligned}
$$

Thermal turbine.

$$
T_{s}=0.4 \mathrm{~s}, T_{l}=5.4 \mathrm{~s}, T_{h}=0.25, k_{l}=0.75, k_{n}=0.25 .
$$




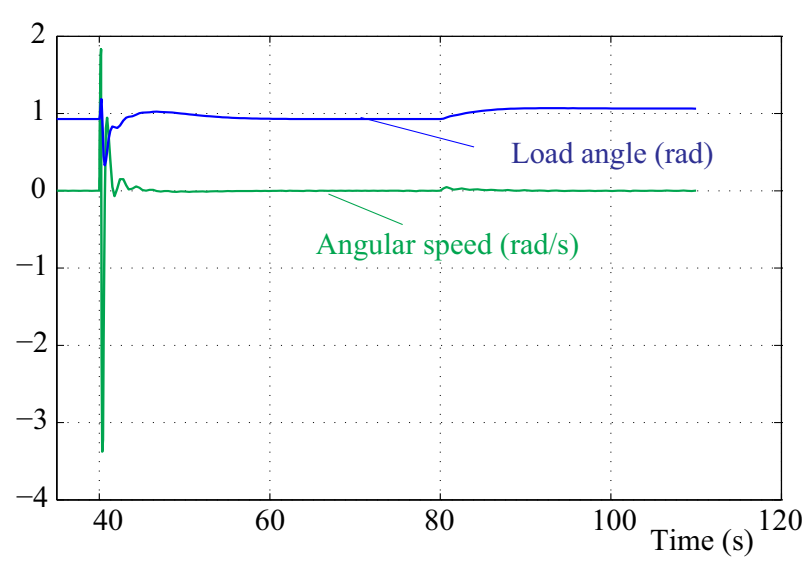

Fig. 1. Dynamic behavior of angular speed and load angle, case $X_{v 3}=2 \mathrm{pu}$

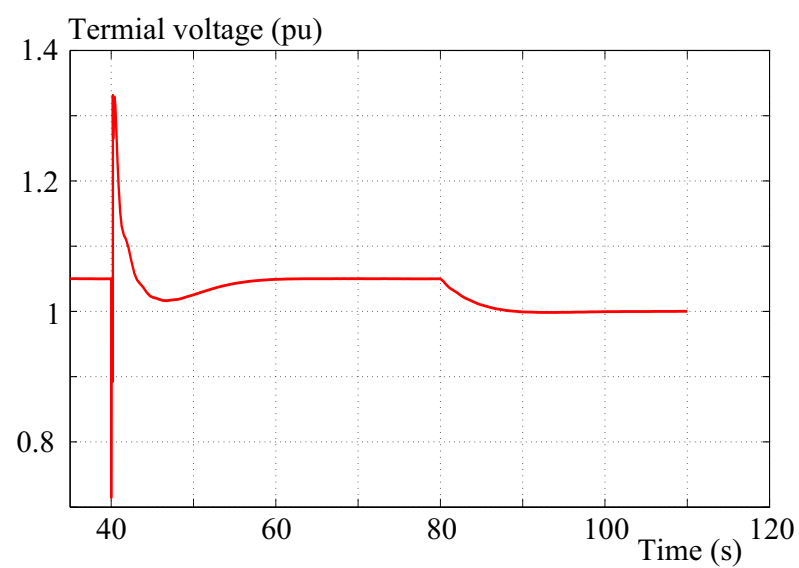

Fig. 3. Dynamic behavior of terminal voltage of SG

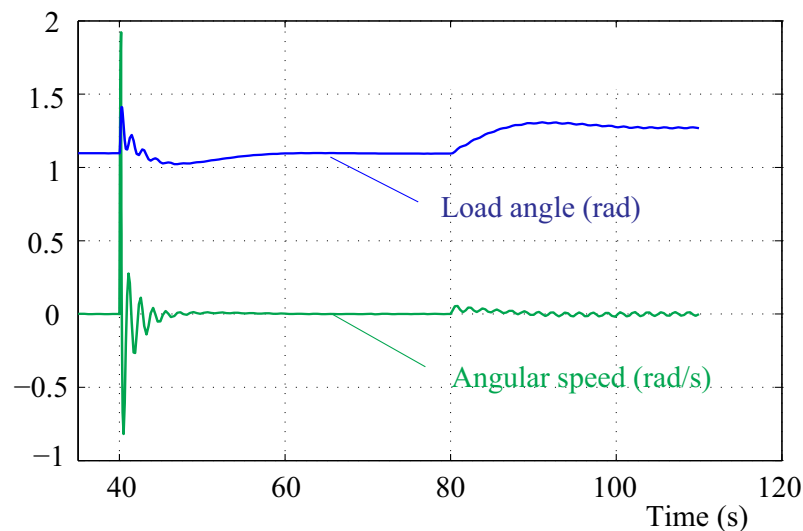

Fig. 5. Dynamic behavior of angular speed and load angle, case $X_{v 3}=0.2 \mathrm{pu}$

Working points are given as $\left(U_{z}, P_{z}\right.$ - set points of terminal voltage and electrical power).

$$
U_{z}=1 \mathrm{pu}, P_{z}=0.9, P=\langle 0.3,0.7,1\rangle .
$$

Performance quality and other parameters

$$
Q_{0}=0.5 * I, Q_{i}=0, S_{0}=0, S_{i}=0, R=I,
$$

$\rho_{i}=10 \mathrm{pu} / \mathrm{s}, i=1,2, \theta_{i} \in\langle-1,1\rangle,\|P(\theta)\| \leq$ ro $=1000$.

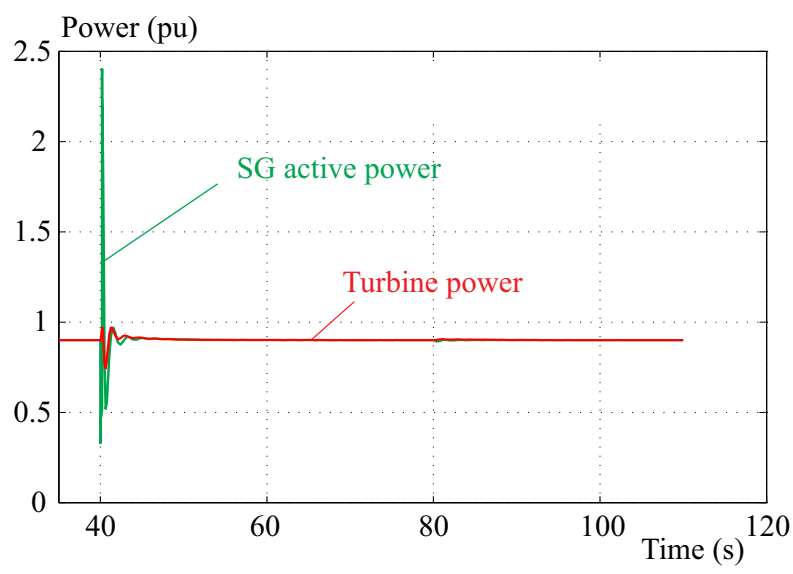

Fig. 2. Dynamic behavior of electrical and turbine power

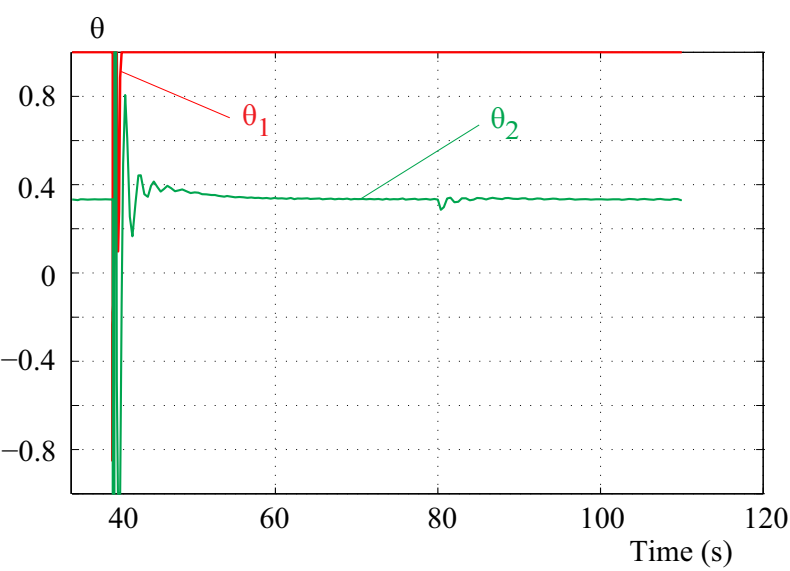

Fig. 4. Dynamic behavior of gain scheduled parameters

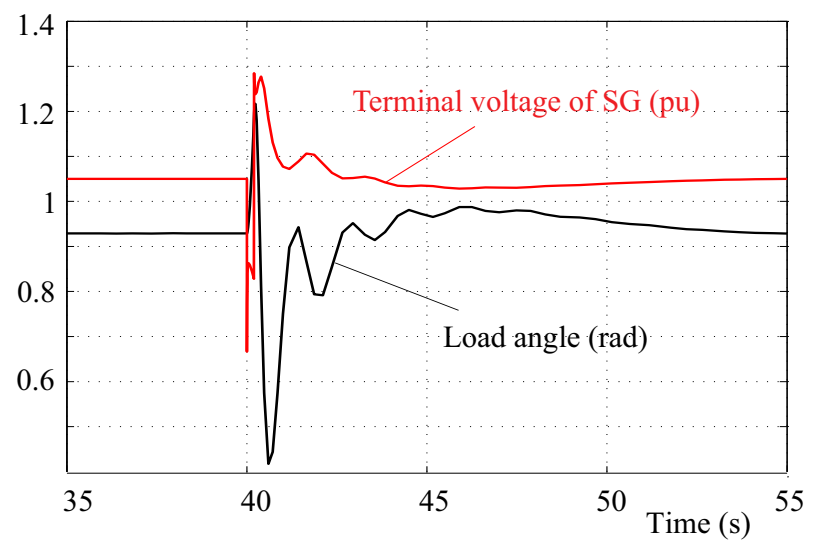

Fig. 6. Dynamic behavior of load angle and terminal voltage with PI controllers

Note that the dimensions of turbogenerator state, input and output matrices are $A \in R^{8 \times 8}, B \in R^{8 \times 2}$, $C \in R^{4 \times 8}, C_{d} \in R^{4 \times 8}$ and output $y=\left[\begin{array}{ll}y_{p} & y_{i}\end{array}\right]$ where $y_{p}, y_{i}$ are output vectors of terminal voltage and active power for proportional and respectively integral of terminal voltage and active power for integral part of controller. Derivative part controller output is $\dot{y}_{p}$. Parameters of designed decentralized PI robust gain scheduled controller for excitation control with terminal volt- 


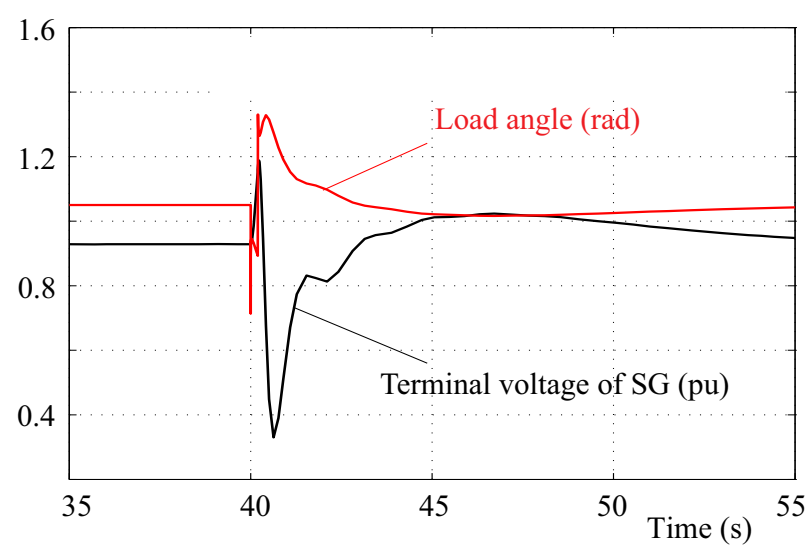

Fig. 7. Dynamic behavior of load angle and terminal voltage with proposed controllers $X_{v 3}=2 \mathrm{pu}$

age feedback .

$$
\begin{aligned}
R_{U}=5.2335+ & \frac{2.5175}{s}- \\
& \left(0.1044+\frac{0.7}{s}\right) \theta_{1}-\left(0.8072+\frac{0.7074}{s}\right) \theta_{2} .
\end{aligned}
$$

Parameters of designed decentralized PI robust gain scheduled controller for governor control.

$$
\begin{aligned}
& R_{T}=3.5512+\frac{0.6671}{s}- \\
& \quad\left(0.0648+\frac{0.1309}{s}\right) \theta_{1}-\left(0.0440+\frac{0.0443}{s}\right) \theta_{2} .
\end{aligned}
$$

Parameters of decentralized power system stabilizer for excitation controller as the first derivative of electrical power.

$$
P S S=-0.6849-0.064 \theta_{1}-0.0316 \theta_{2} .
$$

Above results have been obtained using YALMIP with solver penbmi (free). In this example we assume that

$$
\theta_{j} \in\langle-1,1\rangle, j=1,2 .
$$

Simulation results of nonlinear turbogenerators model with designed controllers $R_{U}, R_{T}$, and $P S S$ proves that the turbogenerator is stable with the performance of all four vertices of uncertain box. The subsequent two simulation experiments are:

- at time $t=40 \mathrm{~s}$ two phase short circuits were realized in the place of system voltage $U_{s}$ when within the time $=0.2 \mathrm{~s}$ the voltage $U_{s}$ shut down from $1 \mathrm{pu}$ to $0.2 \mathrm{pu}$,

- ar time $t=80 \mathrm{~s}$ the terminal voltage set point $U_{z}$ was changed from $1.05 \mathrm{pu}$ to $1 \mathrm{pu}$.

Simulation results of the above two experiments are given in the following figures. In Figs. 1-5 there is the dynamic behavior of many variables of synchronous generator. From the stability point of view, the load angle of SG and terminal voltage play a very important role.
In Figs. 6 and 7 one can see in more detail the dynamic behavior of the above mentioned two synchronous generator variables for classical PI and the paper proposed a robust gain scheduled controller for the case of two phase short circuits.

The above figures imply:

- the performance of closed loop systems for all defined regimes with proposed controllers is less to oscillate than with classical PI ones,

- the proposed controller guarantee the performance, stability and robustness properties of a closed loop system in the given uncertainty box and for all defined SG's regimes,

- the rate of disturbance changes for which the performance, stability and robustness properties are guaranteed could be given by a designer,

- for the disturbances from a given uncertainty box and allowed rates the performance, stability and robustness properties are guaranteed.

\section{CONCLUSIONS}

A unique approach to the design of gain scheduled controller (GSC) is presented. The proposed design procedure is based on the Bellman-Lyapunov equation, guaranteed cost and robust stability conditions using the Parameter Dependent Quadratic Stability approach. The obtained feasible design procedures for robust GSC design are in the form of BMI with guaranteed convex stability conditions. The obtained design results and their properties are illustrated in the simultaneously design of controllers for a simple model (6-order) turbogenerator. The results of the obtained design procedure are a PI automatic voltage regulator (AVR) for synchronous generator, a PI governor controller, and a power system stabilizer for excitation system. All simulation results performed using nonlinear 6-order model of turbogenerator with designed of gain scheduled controllers. The obtained dynamic behavior exhibits that in the paper proposed controllers for all the defined turbogenerator regimes guarantee stability, performance and robustness properties of closed loop system, and give superior performance than classical PI controllers. A very important, factor is that the designer can define the rate of disturbance changes for which the stability and robustness properties need to be ensured. When the rate of turbogenerator parameters changes are known, the $\dot{\theta}$, one can take it account to guarantee the stability of closed loop system with nonlinear model and gain scheduled controller. Theory of large scale system implies that for increasing the stability of large scale system, the performances of all subsystems need to be increased (overshoot need to be decreased). From this point of view investigation of a single machine infinite bus power system gives the way how to possible improve the stability of multimachine power system. Due to proposed relaxed infinite bus model, we suppose that 
multimachine power system with designed controller parameters will be stable and the controller parameters will be near to the optimal value. For practical implementation of proposed gain scheduled controller the controller parameters need to be tuned a such a way that for all outputs (terminal voltage and electrical power) the overshoot need to be decreased without changing the settling time.

\section{Acknowledgment}

The work was supported by Grant 1/0475/16 of the Slovak Grant Agency.

\section{REFERENCES}

[1] T. Lahdhiri, A. T. Alouani, "Nonlear Excitation Control of a Synchronous Generator with Implicit Termal Voltage Regulation", Electric Power Sys. Research, vol. 36, 1996, pp. 101-112.

[2] S. A. Pavlushinko, "Automatic Excitation Control of Synchronous Generators as an Effective Means to Ensure the Reliable Parallel Operation of Generation Equipment and the United Power System as a Whole", Power Technology and Engineerg, vol. 46, no. 5, 2013, pp. 399-406.

[3] J. Alveraz-Ramirez, I. Cervantes, R. Escarela-Perez and G. Espriasa-Perez, "A Two-Loop Excitation Control System for Synchronnous Generators", Electrical Power and Energy Systems, vol. 27, 2005, pp. 556-566.

[4] L. L. Karnavas and P. D. Papadopoulos, "Excitation Control of a Synchronous Machine using Polynomial Neural Networks", Journal of Electrical Engineerg, vol. 55, no. 7-8, 2004, pp. 169-179.

[5] M. Cao, T. L. Shen, Y. H. Song and S. W. Mei, "Nonlinear Disturbance Attenuation Controller for Turbogenerators Power Systems via Recursive Design", : Proc. of the 2002 t. Conf. on Control Applications, University of Grongen, vol. 2, 2002, pp. 938-943.

[6] A. A. Kolesnikov and A. A. Kuzmenko, "Synergetic Synthesis of Nonlinear interconnected Control for Turbogenerators", Taganinrog State University of Radia, June 2002, pp. 1-6.

[7] A. A. Ba-mugabel and M. A. Abido, "Review of Conventional Power System Stabilizer Design Methods", IEEEGCC Conference, March 2006 IE, Manama, pp. 1-7.

[8] E. Ghalipour and S. M. Nosratabadi, "A New Coordation Strategy of SSSC and PSS Controllers on Power System usg SOA Algorithm Based on Pareto Method", Electrical Power and Energy Systems, vol. 67, 2015, pp. 462-471.

[9] P. Zhao, W. Yao, J. Wen, L. Jiang, S. Wang and S. Cheng, "Improved Synergetic Excitation Control for Transient Stability Enhancement and Voltage Regulation of Power Systems", Electrical Power and Energy Systems, vol. 68, 2015, pp. 44-51.

[10] H. Liu, Z. Hu and Y. Song, "Lyapunov-Based Decentralized Excitation Control for Global Asymptotic Stability and Voltage Regulation of Multi-Machine Power Systems", IEEE Trans. on Power Systems, vol. 27, no. 4, 2012, pp. 2262-2270.

[11] P. Kundur, M. Klein, G. J. Rogers and M. S. Zywno, "Application of Power System Stabilizers for Enhancement of Overall System Stability", IEEE Trans. on Power Systems, vol. 4, no. 2, 1989, pp. 614-624.

[12] E. Z. Zhou, O. P. Malik and G. S. Hope, "Design of Stabilizer for a Multimachine Power Systems based on the Sensivity of PSS Effect", IEEE Trans. on Energy Conversion, vol. 7, no. 3, 1992, pp. $606-614$.
[13] S. Dechanupaprittha, I. Ngamroo and Y. Mitani, "Decentralized Design of Robust Power System Stabilizers Considering System Uncertainties,": Power Tech IEEE Russia, 2005, CD-ROM.

14] V. Veselý and A. Kozáková, "Robust PSS Design for a Multivariable Power System", : Power Tech Russia, 2004, CD-ROM.

15] H. Liu, J. Qi, J, Wang and P. Li, "Decentralized Voltage and Power Regulation Control of Excitation and Governor System with Global Asymptotic Stability", Submitted to the IEEE Trans. on Power systems, 2015, arXiv:1509.00421[math.OC].

[16] M. Aldeen and F. Grusca, "Multimachine Power System Stabilizer Design Based on New LQR Approach", IEE Proc. Gener. Trans. Distrib. vol. 142 , no. 5, 1995, pp. 494-502.

[17] V. A. Venikov, a) "Transient Electromechanical Processes Electric Power Systems", Vyssaja skola, Moscow (Russian), 1985. b) "Transient Processes Electrical Power Systems", Mir, Moscow, 1978.

[18] P. Kundur, "Power System Stability and Control", McGraw-Hill, c. New York, 1994.

[19] J. Machovski, J. W. Bialek and J. R. Bumby, "Power System Dynamic Stability and Control", John Wiley and Sons, Ltd, 2008

20] V. Veselý and A. Ilka, "Ga-Scheduled PID Controller Design", Journal of Process Control, vol. 23, 2013, pp. 1141-1148.

21] V. Veselý and D. Rosinová, "Robust PID-PSD Controller Design: BMI Approach", Asian Journal of Control, vol. 15, no. 2, 2013, pp. 469-478.

[22] V. M. Kuncevic and M. M. Lycak, "Control Systems Design usg Lyapunov Function Approach", Nauka, Moskau, 1977, Russian.

23] M. R. Esmaili, A. Khodabakhshian, P. Ghaebi Panah, and S. Azirkhan, "A New Robust Multi-Machine Power System Stabilizer Design using Quantitative Feedback Theory", Procedia Technology vol. 11, 2013, pp. 75-85

[24] A. E. Leona, J. M. Mauriciob and J. A. Solsonaa, "Multi-Machine Power System Stability Improvement usg an ObserverBased Nonlinear Controller", Electric Power Systems Research, vol. 89, 2012, pp. 204-214.

25] A. Khodabakhshian and R. Hemmati, "Robust Decentralized Multi-Machine Power System Stabilizer Design usg Quantitative Feedback Theory", Electrical Power and Energy Systems, vol. 41, 2012, pp. 112-119.

[26] T. Bian, Y. Jiang, and Z. P. Jiang, "Decentralized Adaptive Optimal Control of Large-Scale Systems with Application to Power Systems", IEEE Transactions on dustri, vol. 62, no. 4, 2015, pp. 2439-2447.

[27] Y. Tamura and N. Yoro, "Possibility of Auto-Hetero-Parametric Resonances Power Systems and their Relationship with Long-Term Dynamics", IEEE Trans on Power Systems, vol. PWRS-2, no. 4, Nov 1987, pp. 890-896.

28] D. J. Leith and W. E. Leithead, "Survey of Ga-Schedulg Analysis and Design", International Journal of Control, vol. 73, no. 11, 2000,pp. 1001-1025.

Received 10 February 2017

Vojtech Veselý was born in Velké Kapušany, Slovakia in 1940. He received MSc degree in Electrical Engineering from the Leningrad Electrical Engineering Institute, St. Peterburg, Russia, in 1964, PhD and DSc degrees from the Slovak University of Technology, Bratislava, Slovakia, in 1971 and 1985, respectively. Since 1964 he has been with the Department of Automatic Control Systems, STU FEI in Bratislava. Since 1986 he has been a full professor. His research interests include the areas of power system control, decentralized control of large-scale systems, robust control, predictive control and optimization. He is author or coauthor of more than 300 scientific papers. 